\title{
Preface
}

\section{Cellular Signalling - Special issue to celebrate 75th birthday of Prof. Robert J. Lefkowitz}

G protein-coupled receptors (GPCRs), also known as seven transmembrane receptors (7TMRs), are at the center stage of almost every cellular and physiological process in our body. They recognize a diverse array of signals and communicate the information across the cell membrane to allow the cells to integrate signalling cascades to induce appropriate functional responses. Interestingly, GPCRs display a remarkably conserved architecture harbouring seven transmembrane helices, and highly convergent signaling and regulatory properties. GPCRs have traditionally been, and continue to be the sweethearts of the pharmaceutical industry with more than one third of the marketed drugs targeting these receptors.

Starting from the development of radioligand binding assays and molecular cloning of the $\beta 2$ adrenergic receptor, the laboratory of Prof. Robert J. Lefkowitz (Bob) at the Duke University Medical Center in Durham has been the pioneer in uncovering various paradigms of GPCR desensitization, regulation and signaling. Different phases of research in Bob's laboratory have essentially defined key aspects of receptor-effector coupling, multifaceted roles of GRKs and $\beta$-arrestins, novel cross-talk mechanisms, biased signalling and many more. In addition to these unparalleled research contributions, as Bob himself points out, one of the most satisfying aspects of his career is training a large number of fellows who have gone on to emerge as the leaders in the field of GPCR biology. Most of us who have had the privilege to work with Bob continue to cherish his infectious enthusiasm, charismatic ability to cheer us up all the time, and his intense passion for scientific pursuit.

This special issue of Cellular Signalling is a small tribute to Bob's everlasting contributions in the field of GPCR biology on the occasion of his $75^{\text {th }}$ birthday. This issue contains ten different review articles written by the alumni of the Lefkowitz laboratory, and they cover a wide spectrum of topics ranging from ligand-receptor interactions, receptor-effector interactions, signalling paradigms, desensitization mechanisms, and biased agonism. Many of the Lefkowitz lab alumni who were unable to contribute due to time constraints have sent their appreciative greetings and best wishes for this special issue of Cellular Signalling.

We want to sincerely thank all the authors who have taken time despite their incredibly busy schedule to write wonderful reviews for this special issue. We also extend our sincere gratitude to the journal editorial staff and production team for working relentlessly to put together this special issue in a timely fashion.

Finally, on behalf of all the past and present fellows of the Lefkowitz laboratory - A very Happy 75th Birthday, Bob!!!

Arun K. Shukla*, Nigel J. Pyne

http://dx.doi.org/10.1016/j.cellsig.2017.10.015 * Corresponding author.

Cellular Signalling 41 (2018) 1 Available online 31 October 2017 0898-6568/ (C) 2017 Elsevier Inc. All rights reserved. 